\title{
Remarks on the Pricing of Contingent Claims under Constraints
}

\author{
A. Bensoussan \\ University of Paris-Dauphine \\ Place du Maréchal de Lattre de Tassigny \\ Paris Cedex 16, 75775, France \\ (e-mail: Alain-Bensoussan@wanadoo.fr)
}

November 9, 2003

\section{Introduction}

In this paper we consider the results of I. Karatzas, S.E. Shreve [5] related to the problem of contingent claims with constraints, see also I. Karatzas, S.G. Kou [4]. We propose a different approach, which is more analytic, and also more elementary and straightforward. However, it is limited to the case when the Dynamic Programming approach is possible

Our approach has also the advantage of working without any consumption process, which turns out to be somewhat artificial and slightly confusing.

\section{SETTING OF THE PROBLEM}

\section{$2.1 \quad$ ASSUMPTIONS}

Let

$$
\otimes, \mathcal{A}=\text { Borel } \sigma \text { - algebra on } \Omega, P,
$$

a probability space, equipped with a filtration and a $n$ dimensional standardized Wiener process for this filtration

$$
\mathcal{F}^{s}, w(s) \text {. }
$$


We consider

$b(t)$, bounded adapted process in $R^{n}$

$r(t)$ a bounded continuous deterministic scalar function

and

$$
\begin{aligned}
& \sigma(t) \quad n \times n \text { bounded continuous with bounded inverse matrix; } \\
& a(t)=\sigma(t) \sigma^{*}(t)
\end{aligned}
$$

We consider a market with $n$ stocks, governed by the usual Ito equations

$$
d S_{i}(s)=S_{i}(s)\left(b_{i}(s) d s+\Sigma_{j} \sigma_{i j}(s) d w^{j}\right), i, j=1, \cdots, n, S(0) \geq 0, \neq 0
$$

We set

$$
a(s)=\sigma(s) \sigma(s)^{*}
$$

There is also a risk-free money market, given by

$$
d S_{0}(s)=S_{0}(s) r(s)(s) d s, S_{0}(0)=1
$$

We define

$$
\begin{aligned}
& \gamma_{0}(s)=\frac{1}{S_{0}(s)} \\
& \theta(s)=\sigma^{-1}(s)(b(s)-r(s) \mathbb{I})
\end{aligned}
$$

Since $\theta$ is bounded, we can define a new probability on $\otimes, \mathcal{A}$, by setting

$$
\left.\frac{d P^{0}}{d P}\right|_{\mathcal{F}^{s}}=\exp \left\{-\int_{0}^{s} \theta(\tau) d w(\tau)-\frac{1}{2} \int_{0}^{s}|\theta(\tau)|^{2} d \tau\right\}
$$

From the Girsanov theorem, if we introduce the process

$$
w^{0}(s)=w(s)+\int_{0}^{s} \theta(\tau) d \tau,
$$

then the system $\Omega, \mathcal{A}, \mathcal{F}^{s}, P_{0}, w^{0}(s)$ forms a probability system in which $w^{0}(s)$ is a $\mathcal{F}^{s}$ standardized Wiener process. Note that from (2.4) one has :

$$
d S_{i}(s)=S_{i}(s)\left(r(s) d s+\Sigma_{j} \sigma_{i j}(s) d w_{j}^{0}\right), i, j=1, \cdots, n
$$


A portfolio of assets is a vector of adapted processes

$$
\pi_{0}(s), \pi(s)=\left(\pi_{1}(s), \cdots, \pi_{n}(s)\right)
$$

such that

$$
\int_{0}^{T}\|\pi(s)\|^{2} d s<+\infty, \quad \text { a.s. }
$$

We next define the Wealth as the process

$$
X(s)=\sum_{i=0}^{n} \pi_{i}(s)
$$

We assume the self financing property, which amounts to the fact that the Wealth process has the following Ito differential

$$
d X(s)=\sum_{i=0}^{n} \pi_{i}(s) \frac{d S_{i}(s)}{S_{i}(s)}
$$

and, as well known, using (2.11)

$$
d X(s)=r(s) X(s) d s+\Sigma_{i, j} \pi_{i} \sigma_{i j}(s) d w_{j}^{0}
$$

The initial wealth will be given by

$$
X(0)=x
$$

We shall denote by

$$
X_{x}^{\pi}(s)
$$

the Wealth process, corresponding to an initial Wealth $x$ and a portfolio $\pi$ We define the set of portfolios

$$
\begin{aligned}
& A=\left\{\pi \mid \pi \text { adapted, } \int_{0}^{T}\|\pi(s)\|^{2} d s<+\infty, \quad\right. \text { a.s. } \\
& \left.X_{x}^{\pi}(s) \geq-\Lambda_{x}^{\pi}, \forall s \in[0, T], \Lambda_{x}^{\pi} \geq 0, E^{0} \Lambda_{x}^{\pi}<\infty, 1<p<+\infty\right\}
\end{aligned}
$$

We note that the set $A$ does not depend on $x$, which explains the notation. It is easy to check that, whenever $\pi \in A$, the process $X_{x}^{\pi}(s) \gamma_{0}(s)$ is a $\mathcal{F}^{s}, P^{0}$ supermartingale, and in particular

$$
E^{0} X_{x}^{\pi}(s) \gamma_{0}(s) \leq x, \forall s \in[0, T]
$$




\subsection{CONTINGENT CLAIMS}

Let $h(S)$ be a function such that

$$
h \text { continuous, } 0 \leq h(S) \leq C\left(1+|S|^{\beta_{0}}\right), \forall S \geq 0, h(S)>0, \forall S \geq 0, \neq 0
$$

A Contingent Claim is the variable

$$
B=h(S(T))
$$

which, thanks to the assumption (2.20) and (2.4) is a.s. strictly positive and $\mathcal{F}^{T}$ measurable. The concept of arbitrage opportunity is the following: we say that a price $u$ represents an opportunity of arbitrage in selling, if $\exists \quad x<0, x+u>0$ such that $\exists \pi \in A$, with

$$
X_{x+u}^{\pi}(T) \geq B \text { a.s. }
$$

Similarly, we say that a price $u$ represents an opportunity of arbitrage in buying, if $\exists x<0$ such that $\exists \pi \in A$, with

$$
X_{x-u}^{\pi}(T)+B \geq 0 \text { a.s. }
$$

A price presents an arbitrage opportunity if it presents an opportunity of arbitrage in selling or in buying. An opportunity of arbitrage is a very favourable situation, since it leads to the possibility of an infinite wealth. Indeed, consider the selling case, for instance, we sell $k>1$ contingent claims, and use the portfolio

$$
\tilde{\pi}=\frac{x+k u}{x+u} \pi
$$

which also belongs to $A$ and keeps the same proportion of wealth for each stock, then one has

$$
X_{x+k u}^{\tilde{\pi}}(T)-k B=\frac{x+k u}{x+u}\left(X_{x+u}^{\pi}(T)-B\right)+\frac{(1-k) x}{x+u} B \geq \frac{(1-k) x}{x+u} B
$$

and since $x<0$, this tends to $+\infty$ as $k \rightarrow \infty$. 


\subsection{CONSTRAINTS}

Let $K_{+}, K_{-}$be two non empty convex closed subsets of $R^{n}$. We shall write $K$ for $K_{+}$or $K_{-}$, to simplify the notation, whenever there is no confusion. We set for $x \geq 0$

$$
A\left(x, K_{+}\right)=\left\{\begin{array}{l}
\pi \in A, \Lambda_{x}^{\pi}=0, p(s)=\frac{\pi(s)}{X_{x}^{\pi}(s)} \in K_{+}, \text {if } X_{x}^{\pi}(s)>0 \\
\pi(s)=0, \text { if } X_{x}^{\pi}(s)=0
\end{array}\right.
$$

Suppose there exists $\pi \in A\left(x, K_{+}\right)$such that

$$
X_{x}^{\pi}(T) \geq B \text { a.s. }
$$

then for $x^{\prime} \geq x$ one considers $X_{x^{\prime}}^{\pi^{\prime}}(s)$ with

$$
\pi^{\prime}(s)=\frac{x^{\prime}}{x} \pi(s)
$$

and clearly

$$
X_{x^{\prime}}^{\pi^{\prime}}(s)=\frac{x^{\prime}}{x} X_{x}^{\pi}(s)
$$

Therefore

$$
\pi^{\prime} \in A\left(x^{\prime}, K_{+}\right)
$$

and

$$
X_{x^{\prime}}^{\pi^{\prime}}(T) \geq B \text { a.s. }
$$

This leads to the definition

$$
h_{u p}\left(K_{+}\right)=\inf \left\{x \geq 0 \mid \exists \pi \in A\left(x, K_{+}\right) \quad \text { with } X_{x}^{\pi}(T) \geq B \text { a.s. }\right\}
$$

Remark 2.1. Because of (2.19), one cannot hope to have (2.26) with $x<0$, even without the constraint

The quantity $h_{u p}\left(K_{+}\right)$is called the upper hedging price.

We proceed similarly to define the lower hedging price.

We set for $x \geq 0$

$$
A\left(x, K_{-}\right)=\left\{\begin{array}{l}
\pi \in A, X_{-x}^{\pi}(s) \leq 0, p(s)=\frac{\pi(s)}{X_{-x}^{\pi}(s)} \in K_{-}, \text {if } X_{-x}^{\pi}(s)<0 \\
\pi(s)=0, \text { if } X_{-x}^{\pi}(s)=0
\end{array}\right.
$$


Suppose there exists $\pi \in A\left(x, K_{-}\right)$such that

$$
X_{-x}^{\pi}(T)+B \geq 0 \text { a.s. }
$$

Considering $x^{\prime}<x$, and again

$$
\pi^{\prime}(s)=\frac{x^{\prime}}{x} \pi(s)
$$

then

$$
X_{-x^{\prime}}^{\pi^{\prime}}(s)=\frac{x^{\prime}}{x} X_{-x}^{\pi}(s)
$$

Therefore, necessarily

$$
\pi^{\prime} \in A\left(x^{\prime}, K_{-}\right)
$$

and

$$
X_{-x^{\prime}}^{\pi^{\prime}}(T)+B \geq 0 \text { a.s. }
$$

This leads to the definition

$$
h_{\text {low }}\left(K_{-}\right)=\sup \left\{x \geq 0 \mid \exists \pi \in A\left(x, K_{-}\right) \quad \text { with } X_{-x}^{\pi}(T)+B \geq 0 \text { a.s. }\right\}
$$

Remark 2.2. Note that $0 \in A\left(0, K_{-}\right)$, and satisfies the condition (2.30).

The quantity $h_{\text {low }}\left(K_{-}\right)$is called the lower hedging price. We shall check the elementary property

Lemma 2.3. We have the property

$$
h_{\text {low }}\left(K_{-}\right) \leq h_{\text {up }}\left(K_{+}\right)
$$

\section{PROOF:}

We may assume

$$
h_{\text {low }}\left(K_{-}\right)>0 ; \quad h_{u p}\left(K_{+}\right)<+\infty
$$

otherwise the property is trivial. If there exists

$$
\pi \in A\left(x, K_{+}\right) \quad \text { with } X_{x}^{\pi}(T) \geq B \text { a.s. }
$$

then, using the supermartingale property (2.19), we have

$$
x \geq E^{0} X_{x}^{\pi}(T) \gamma_{0}(T) \geq E^{0} B \gamma_{0}(T) .
$$


Setting

$$
u_{0}=E^{0} B \gamma_{0}(T)
$$

then we deduce

$$
h_{u p}\left(K_{+}\right) \geq u_{0}
$$

and $u_{0}$ is finite. Next, considering $\epsilon$, with

$$
\epsilon<h_{\text {low }}\left(K_{-}\right)
$$

then, setting $x_{\epsilon}=h_{\text {low }}\left(K_{-}\right)-\epsilon$

$$
\exists \pi \in A\left(x_{\epsilon}, K_{-}\right) \quad \text { with } X_{-x_{\epsilon}}^{\pi}(T)+B \geq 0
$$

and from the supermartingale property again,we get

$$
-x_{\epsilon} \geq E^{0} X_{-x_{\epsilon}}^{\pi}(T) \gamma_{0}(T) \geq-E^{0} B \gamma_{0}(T)
$$

therefore

$$
x_{\epsilon} \leq u_{0}
$$

and letting $\epsilon$ tend to zero, we obtain :

$$
h_{\text {low }}\left(K_{-}\right) \leq u_{0}
$$

which, compared to (2.32)completes the proof.

Remark 2.4. It is an immediate consequence of the definitions that a price $u>h_{u p}\left(K_{+}\right)$leads to an opportunity of arbitrage in selling, and a positive price $u<h_{\text {low }}\left(K_{-}\right)$leads to an opportunity of arbitrage in buying. On the other hand a price $u \in\left[h_{\text {low }}\left(K_{-}\right), h_{u p}\left(K_{+}\right)\right]$cannot offer any opportunity of arbitrage. This interval is called the arbitrage-free interval. 


\section{CHARACTERIZATION OF THE UPPER HEDGING PRICE}

\subsection{STOCHASTIC CONTROL PROBLEM}

We shall consider the upper hedging price, and thus to simplify the notation, we write

$$
K=K_{+}
$$

Let us set

$$
\delta(\nu)=\sup _{\{p \in K\}}\left(-p^{*} \nu\right)
$$

which is a lower semicontinuous (lsc), proper, convex function, finite on its effective domain

$$
\tilde{K}=\{\nu \mid \delta(\nu)<+\infty\}
$$

Using R.T. ROCKAFELLAR [7], the set $\tilde{K}$ is a convex cone, and

$$
0 \in \tilde{K}, \text { with } \delta(0)=0 .
$$

Moreover $\delta$ is positively homogeneous, i.e.

$$
\delta(\lambda \nu)=\lambda \delta(\nu), \text { if } \quad \lambda \geq 0
$$

and subadditive, i.e.

$$
\delta(\nu+\mu) \leq \delta(\nu)+\delta(\mu), \forall \nu, \mu \text {. }
$$

Relying on ROCKAFELLAR again, we state the property

$$
p \in K \Leftrightarrow p^{*} \nu+\delta(\nu) \geq 0, \forall \nu \in \tilde{K}
$$

In the sequel, we shall assume

$$
\delta(\nu) \geq \delta_{0}, \forall \nu
$$

Consider the Hilbert space

$$
L_{\mathcal{F}}^{2}\left(0, T ; R^{n}\right)
$$


of stochastic processes $\nu(s)$ adapted to the filtration $\mathcal{F}^{s}$ such that

$$
E^{0} \int_{0}^{T}\|\nu(s)\|^{2} d s<+\infty .
$$

We then define

$$
\mathcal{D}=\left\{\nu(.) \in L_{\mathcal{F}}^{2}\left(0, T ; R^{n}\right)\left|\nu(s, \omega) \in \tilde{K}, \int_{0}^{T}\right| \delta(\nu(s)) \mid d s<+\infty \text { a.s. }\right\}
$$

Let next, writing $\nu$ for $\nu($.

$$
\begin{aligned}
& Z_{\nu}(s)=\exp \left[-\int_{0}^{s} \sigma^{-1} \nu(\tau) d w^{0}(\tau)-\frac{1}{2} \int_{0}^{s}\left|\sigma^{-1} \nu(\tau)\right|^{2} d \tau\right] \\
& \gamma_{\nu}(s)=\exp \left[-\int_{0}^{s}(r(\tau)+\delta(\nu(\tau))) d \tau\right]
\end{aligned}
$$

These two processes are well defined for any $\nu(.) \in \mathcal{D}$. We set

$$
\mathcal{D}^{(m)}=\left\{\nu(.) \in \mathcal{D} \mid Z_{\nu}(.) \text { is a martingale for } P^{0}, \mathcal{F}^{s}\right\}
$$

It is well known that if

$$
\sup |\nu(s, \omega)|<+\infty
$$

then $\nu(.) \in \mathcal{D}^{(m)}$. We call

$$
\mathcal{D}^{(b)}=\{\nu(.) \in \mathcal{D} \quad \text { bounded }\}
$$

The set $\mathcal{D}^{(b)}$, hence also $\mathcal{D}^{(m)}$ is dense in $\mathcal{D}$. Indeed, we can approximate $\nu($. by

$$
\nu_{k}(s)=\mid \begin{aligned}
& \nu(s) \text { if }|\nu(s)| \leq k \\
& \frac{k}{|\nu(s)|} \nu(s) \text { if }|\nu(s)| \geq k
\end{aligned}
$$

Since

$$
\delta\left(\nu_{k}(s)\right)=\mid \begin{aligned}
& \delta(\nu(s)) \text { if }|\nu(s)| \leq k \\
& \frac{k}{|\nu(s)|} \delta(\nu(s)) \text { if }|\nu(s)| \geq k
\end{aligned}
$$


then clearly $\nu_{k}($.$) belongs to \mathcal{D}^{(b)}$ if $\nu($.$) belongs to \mathcal{D}$. Moreover

$$
\begin{aligned}
& \nu_{k}(.) \rightarrow \nu(.) \in L^{2}\left(0, T ; R^{n}\right), \quad \text { a.s. as } k \rightarrow \infty . \\
& \delta\left(\nu_{k}(.)\right) \rightarrow \delta(\nu(.)) \in L^{1}\left(0, T ; R^{n}\right), \quad \text { a.s. as } k \rightarrow \infty .
\end{aligned}
$$

We shall call in the sequel

$$
H_{\nu}(s)=Z_{\nu}(s) \gamma_{\nu}(s)
$$

and we define

$$
\begin{aligned}
& u_{\nu}=E^{0}\left(H_{\nu}(T) B\right) \\
& \hat{u}=\sup _{\nu(.) \in \mathcal{D}} u_{\nu}
\end{aligned}
$$

If $\nu()=$.0 , we recover the definition (2.31).

We state the easy result

Lemma 3.1. We have the property

$$
\hat{u}=\sup _{\nu(.) \in \mathcal{D}^{(b)}} u_{\nu}=\sup _{\nu(.) \in \mathcal{D}^{(m)}} u_{\nu}
$$

\section{PROOF:}

Clearly

$$
\hat{u} \geq \sup _{\nu(.) \in \mathcal{D}^{(b)}} u_{\nu}
$$

On the other hand, if for any $\nu \in \mathcal{D}$, we consider the approximation $\nu_{k}$, then thanks to (3.13),(3.14), we can state that

$$
H_{\nu_{k}}(T) \rightarrow H_{\nu}(T), \quad \text { a.s. }
$$

and thus, by Fatou's Lemma

$$
E^{0}\left(H_{\nu}(T) B\right) \leq \liminf _{k \rightarrow \infty} E^{0}\left(H_{\nu_{k}}(T) B\right) \leq \sup _{\nu(.) \in \mathcal{D}^{(b)}} u_{\nu}
$$

which implies

$$
\hat{u} \leq \sup _{\nu(.) \in \mathcal{D}^{(b)}} u_{\nu}
$$

and with (3.18) the result. 


\subsection{THE MAIN RESULT}

The main result is the following

Theorem 3.2. Assuming (3.4), (2.20), and then one has

$$
h_{u p}(K)=\hat{u}
$$

We begin by proving that

$$
h_{u p}(K) \geq \hat{u}
$$

PROOF of (3.20)

If we consider $\nu \in \mathcal{D}^{(m)}$, then, since $Z_{\nu}(s)$ is a $P^{0}, \mathcal{F}^{s}$ martingale, then we can define a new probability measure $P^{\nu}$ on $\otimes, \mathcal{A}$, by setting

$$
\left.\frac{d P^{\nu}}{d P^{0}}\right|_{\mathcal{F}^{s}}=Z_{\nu}(s)
$$

From the Girsanov theorem, if we introduce the process

$$
w^{\nu}(s)=w^{0}(s)+\int_{0}^{s} \sigma^{-1} \nu(\tau) d \tau
$$

then the system $\Omega, \mathcal{A}, \mathcal{F}^{s}, P^{\nu}, w^{\nu}(s)$ forms a probability system in which $w^{\nu}(s)$ is a $\mathcal{F}^{s}$ standardized Wiener process. Note that from (2.11) one has:

$$
d S_{i}(s)=S_{i}(s)\left(\left(r(s)-\nu_{i}\right) d s+\Sigma_{j} \sigma_{i j}(s) d w_{j}^{\nu}\right), i, j=1, \cdots, n
$$

We can then write

$$
u_{\nu}=E^{\nu}\left(\gamma_{\nu}(T) B\right)
$$

Suppose now that there exists, for $x \geq 0$

$$
\pi \in A(x, K) \quad \text { with } X_{x}^{\pi}(T) \geq B \text { a.s. }
$$

then , using Ito's formula one has

$$
\begin{gathered}
d\left(X_{x}^{\pi}(s) \gamma_{\nu}(s)\right)=\gamma_{\nu}(s) \pi^{*}(s) \sigma(s) d w^{\nu}- \\
-\gamma_{\nu}(s) X_{x}^{\pi}(s)\left(\frac{\pi^{*}(s)}{X_{x}^{\pi}(s)} \nu(s)+\delta(\nu(s))\right)
\end{gathered}
$$


and from (3.3)

$$
\leq \gamma_{\nu}(s) \pi^{*}(s) \sigma(s) d w^{\nu}
$$

It follows easily that

$$
x \geq E^{\nu}\left(X_{x}^{\pi}(T) \gamma_{\nu}(T)\right) \geq E^{\nu}\left(B \gamma_{\nu}(T)\right) .
$$

Therefore we have

$$
x \geq u_{\nu}
$$

and since $\nu$ is arbitrary, we obtain (3.20).

We next introduce the function

$$
u(S, t)=u(S, t ; h)=E^{0} h\left(S_{S, t}(T)\right) \gamma_{0}(t, T)
$$

where $S_{S, t}(T)$ represents the solution of (2.11) with initial data $S$ at time $t$, namely

$$
S_{i, S, t}(T)=S_{i} \exp \left\{\int_{t}^{T}\left(r(s)-\frac{1}{2} a_{i i}(s)\right) d s+\int_{t}^{T} \Sigma_{j} \sigma_{i j}(s) d w_{j}^{0}\right\}
$$

and

$$
\gamma_{0}(t, T)=\frac{\gamma_{0}(T)}{\gamma_{0}(t)}
$$

Clearly, we have (Markov property)

$$
S_{S, 0}(T)=S_{S(t), t}(T)
$$

and

$$
u(S, 0)=u_{0}
$$

It is useful to note the following change of variables: $S_{i}=\exp y_{i}$, and

$$
v(y, t)=v(y, t ; h)=u\left(\cdots, \exp y_{i}, \cdots, t\right)
$$

with

$$
\begin{aligned}
& \frac{\partial v}{\partial t}-r(t) v+\left(r(t)-\frac{1}{2} a_{i i}(t)\right) \frac{\partial v}{\partial y_{i}}+\frac{1}{2} a_{i, j}(t) \frac{\partial^{2} v}{\partial y_{i} \partial y_{j}}=0 \\
& v(y, T)=h\left(\cdots, \exp y_{i}, \cdots\right)
\end{aligned}
$$




\section{PROOFS}

\subsection{PRELIMINARIES}

We shall use results related to the Cauchy problem (3.31). More precisely, we consider the problem

$$
\begin{aligned}
& \frac{\partial z}{\partial t}-r(t) z+\left(r(t)-\frac{1}{2} a_{i i}(t)\right) \frac{\partial z}{\partial y_{i}}+\frac{1}{2} a_{i j}(t) \frac{\partial^{2} z}{\partial y_{i} \partial y_{j}}=0 \\
& z(y, T)=\phi(y)
\end{aligned}
$$

where the initial condition $\phi(y)$ satisfies

$$
\phi \text { measurable }, 0 \leq \phi(y) \leq c\left(1+\exp \beta_{0}|y|\right), \quad \text { a.e. }
$$

Introduce the functional spaces

$$
\begin{aligned}
& L_{\beta}^{2}=L_{\beta}^{2}\left(R^{n}\right)=\left\{\psi(y) \exp -\beta|y| \in L^{2}\left(R^{n}\right)\right\} \\
& H_{\beta}^{1}\left(R^{n}\right)=\left\{\psi \in L_{\beta}^{2} \mid D \psi \in L_{\beta}^{2}\right\} \\
& W_{\beta}(0, T)=\left\{\psi(y, t) \mid \psi \in L^{2}\left(0, T ; H_{\beta}^{1}\right), \frac{\partial \psi}{\partial t} \in L^{2}\left(0, T ; H_{\beta}^{-1}\right)\right\}
\end{aligned}
$$

where $H_{\beta}^{-1}$ represents the dual of $H_{\beta}^{1}$.

From J.L. LIONS [6], it follows that

$$
W_{\beta}(0, T) \subset C^{0}\left([0, T] ; L_{\beta}^{2}\right)
$$

with continuous injection.

The function $\phi(y)$ belongs to $L_{\beta}^{2}$,for any $\beta>\beta_{0}$. We recall the equations (3.29), and introduce the stochastic processes

$$
y_{i, y, t}(s)=y_{i}+\int_{t}^{s}\left(r(\tau)-\frac{1}{2} a_{i i}(\tau)\right) d \tau+\int_{t}^{s} \Sigma_{j} \sigma_{i, j}(\tau) d w_{j}^{0}
$$

We denote by

$$
y_{y, t}(s) \text { the solution of }(4.5)
$$

and to simplify the notation write $\rho(t, s)$ for the vector of coordinates

$$
\rho_{i}(t, s)=\int_{t}^{s}\left(r(\tau)-\frac{1}{2} a_{i i}(\tau)\right) d \tau .
$$

We state the 
Theorem 4.1. Assuming (2.2), (2.3), (4.2), there exists one and only one solution of (4.1), $z \in W_{\beta}(0, T)$, for any $\beta>\beta_{0}$. Moreover,

$$
\begin{aligned}
& z, D z, D^{2} z, \frac{\partial z}{\partial t} \text { are continuous on } R^{n} \times[0, T) \quad ; z(y, t) \geq 0 . \\
& 0 \leq z(y, t) \leq C\left(1+\exp \beta_{0}|y|\right), \forall y, t \\
& \phi \text { is continuous }
\end{aligned}
$$

If

then one has

$$
z \text { is continuous on } R^{n} \times[0, T] \text {. }
$$

One has the probabilistic interpretation

$$
z(y, t)=E^{0} \phi\left(y_{y, t}(T)\right) \gamma_{0}(t, T)
$$

\section{PROOF:}

We do not detail everything, and refer to classical results, see A. FRIEDMAN [2]. Whenever (4.8) holds, then we have (4.6) and (4.1)holds on $R^{n} \times[0, T)$. Moreover, we have (4.9), and the probabilistic interpretation (4.10).

We proceed with a priori estimates. We test (4.1) with $z \exp -2 \beta|y|, \beta>\beta_{0}$, and integrate over $R^{n}$. We obtain easily the estimate

$$
\|z\|_{\left\{W_{\beta}(0, T)\right\}} \leq C_{\beta}\|\phi\|_{\left\{L_{\beta}^{2}\right\}}
$$

and thus also, from (4.4)

$$
\|z\|_{\left\{C^{0}\left([0, T] ; L_{\beta}^{2}\right)\right\}} \leq C_{\beta}\|\phi\|_{\left\{L_{\beta}^{2}\right\}}=
$$

To extend the results when (4.8) is no longer valid, we first notice, from the variational theory of linear parbolic P.D.E. of J. L. LIONS [4], that there exists one and only one solution of (4.1), $z \in W_{\beta}(0, T)$, and $z \geq 0$. The estimates (4.11) and (4.12) still hold. We can then consider independently, the function denoted for the time being by

$$
\zeta(y, t)=E^{0} \phi\left(y_{y, t}(T)\right) \gamma_{0}(t, T)
$$


which makes sense, even when $\phi$ is not continuous. From the relation (4.5) and the notation hereafter, we have

$$
y_{y, t}(T)=y+\rho(t, T)+\int_{t}^{T} \sigma d w^{0} .
$$

Let

$$
\Gamma(t, T)=\int_{t}^{T} a(\tau) d \tau
$$

Since $y_{y, t}(T)$ is gaussian, with mean $y+\rho(t, T)$ and covariance matrix $\Gamma(t, T)$, we can write the formula

$\zeta(y, t)=\int_{R^{n}} \phi(x) \frac{\exp \left[-\frac{1}{2} \Gamma(t, T)^{-1}(x-y-\rho(t, T)) \cdot(x-y-\rho(t, T))\right]}{(2 \pi)^{\frac{n}{2}}|\Gamma(t, T)|^{\frac{1}{2}}} d x \gamma_{0}(t, T)$

Performing easy majorations, one gets, for all $\beta^{\prime}>\beta_{0}$ the inequality

$$
\zeta(y, t) \leq\|\phi\|_{\left\{L_{\beta^{\prime}}^{2}\right\}}\left(\exp \beta^{\prime}|y|\right) K(t, T)
$$

where

$$
K(t, T)=\exp \beta^{\prime}|\rho(t, T)| \frac{\left(\int_{R^{n}} \exp \left[-|\eta|^{2}+2 \beta^{\prime}\left|\Gamma(t, T)^{\frac{1}{2}} \eta\right|\right] \quad d \eta\right)^{\frac{1}{2}}}{(2 \pi)^{\frac{n}{2}}|\Gamma(t, T)|^{\frac{1}{4}}} \gamma_{0}(t, T)
$$

and chosing $\beta>\beta^{\prime}$, we obtain, after easy calculations

$$
\|\zeta(., t)\|_{L_{\beta}^{2}} \leq\|\phi\|_{\left\{L_{\beta^{\prime}}^{2}\right\}} K(t, T)\left(\frac{(n-1) !}{\left(2\left(\beta-\beta^{\prime}\right)\right)^{n-1}}\right)^{\frac{1}{2}}
$$

We next show that, for any $\phi$, satisfying (4.2), there exists a sequence of continuous functions $\phi_{\mu}$, such that

$$
0 \leq \phi_{\mu}(y) \leq c\left(1+\exp \beta_{0}|y|\right)
$$

and for any $\beta>\beta_{0}$, and for any $\epsilon$, there exists $\mu_{\beta}(\epsilon)$, with

$$
\| \phi-\left.\phi_{\mu_{\beta}(\epsilon)}\right|_{L_{\beta}^{2}} \leq \epsilon
$$


This is done by the classical procedure of cutting and regularization. Let $B_{R}$ be the ball of center 0 and radius $R$. The function

$$
\phi_{R}(y)=\frac{\phi(y) \mathbb{1}_{B_{R}}}{1+\exp \beta_{0}|y|}
$$

is positive, bounded by $c$, and vanishes outside $B_{R}$. Clearly

$$
\phi \mathbb{I}_{B_{R}} \rightarrow \phi \text { in } L_{\beta}^{2} \text {, as } R \rightarrow+\infty \text {. }
$$

Consider next the smoothing procedure by the function

$$
\theta_{\lambda}(y)=\frac{1}{\lambda^{n}} \theta\left(\frac{y}{\lambda}\right)
$$

with

$$
\theta(y)=\mid \begin{aligned}
& \frac{1}{a} \exp -\frac{1}{1-|y|^{2}} \text { if }|y| \leq 1 \\
& 0 \text { if }|y| \geq 1
\end{aligned}
$$

and the scalar $a$ is such that

$$
\int_{R^{n}} \theta(y) d y=1
$$

For each fixed $R$, the function

$$
\left(1+\exp \beta_{0}|y|\right) \theta_{\lambda} * \phi_{R} \rightarrow \phi \mathbb{1}_{B_{R}} \text { in } L^{2}\left(R^{n}\right) \text { as } \lambda \rightarrow 0 .
$$

Set

$$
\mu=(\lambda, R)
$$

and

$$
\phi_{\mu}(y)=\left(1+\exp \beta_{0}|y|\right) \theta_{\lambda} * \phi_{R}(y) .
$$

Then $\phi_{\mu}$ is continuous, and recalling that $\phi_{R}(y) \leq c$

$$
\phi_{\mu}(y) \leq c\left(1+\exp \beta_{0}|y|\right) .
$$

Moreover, it is easy to check, that by choosing first $R$, then $\lambda$, one can find $\mu_{\beta}(\epsilon)$ such that (4.18) holds.

These approximations allow us to assert that the probabilistic representation (4.10) still holds, even when the initial condition $\phi$ is not continuous. Indeed, 
let $z_{\mu}, \zeta_{\mu}$ be the functions corresponding to $\phi_{\mu}$, in (4.1) and (4.13). Then, since the probabilistic representation holds for $\phi_{\mu}$, we have

$$
z_{\mu}=\zeta_{\mu}
$$

Also, from (4.12) and (4.17) we have

$$
\left\|z-z_{\mu}\right\|_{L_{\beta}^{2}} \leq C_{\beta}\left\|\phi-\phi_{\mu}\right\|_{L_{\beta}^{2}}
$$

and

$$
\left\|\zeta(., t)-\zeta_{\mu}(., t)\right\|_{L_{\beta}^{2}} \leq C_{\beta, \beta^{\prime}, t}\left\|\phi-\phi_{\mu}\right\|_{L_{\beta^{\prime}}^{2}}
$$

Collecting results, the property (4.10) follows.

From this probabilistic representation, and the growth assumption (4.2), the growth on $z,(4.7)$ follows. The regularity properties (4.6) follow also from the representation formula, and (4.14).

The proof has been completed.

In spite of the fact that $\phi$ is not continuous, we have the following important continuity property. Let us write

$$
y(t)=y_{y, 0}(t)
$$

then we state the following result

\section{Corollary 4.2.}

$$
z(y(t), t) \rightarrow \phi(y(T)), \text { as } t \rightarrow T, \text { in } L^{2}\left(\Omega, \mathcal{A}, P^{0}\right) .
$$

\section{PROOF:}

We first notice that

$$
z(y(t), t)=E^{0}\left[\phi(y(T)) \mid \mathcal{F}^{t}\right] \gamma_{0}(t, T)
$$

which follows from the Markov property

$$
y(T)=y_{y(t), t}(T)
$$


and the representation formula (4.10). Hence $z(y(t), t) \gamma_{0}(t)$ is an $\mathcal{F}^{t}, P^{0}$ martingale. Therefore, from classical martingale estimates, see N. IKEDA, S. WATANABE [3], we have

$$
E^{0} \sup _{\{0 \leq t \leq T\}}\left(|z(y(t), t)| \gamma_{0}(t)\right)^{2} \leq 4 E^{0}\left(\phi(y(T)) \gamma_{0}(T)\right)^{2}
$$

and, since $y(T)$ is gaussian, see also (4.14), we get easily

$$
E^{0} \sup _{\{0 \leq t \leq T\}}|z(y(t), t)|^{2} \leq C_{T, \beta}\|\phi\|_{L_{\beta}^{2}}^{2}
$$

Considering the continuous approximation $\phi_{\mu}$ of $\phi$, and using (4.23), we can write the inequality

$$
E^{0}|z(y(t), t)-\phi(y(T))|^{2} \leq C_{T, \beta}|| \phi-\phi_{\mu}||_{L_{\beta}^{2}}^{2}+3 E^{0}\left|z_{\mu}(y(t), t)-\phi_{\mu}(y(T))\right|^{2} .
$$

Since $z_{\mu}(y, t)$ is continuous on $R^{n} \times[0, T]$, we have for fixed $\mu$, and thanks to $(4.23)$

$$
E^{0}\left|z_{\mu}(y(t), t)-\phi_{\mu}(y(T))\right|^{2} \rightarrow 0, \text { as } t \uparrow T .
$$

The result follows immediately.

\subsection{ADDITIONAL ASSUMPTIONS and COMPLE- TION of PROOFS}

We introduce the function

$$
\hat{h}(S)=\sup _{\{\nu \in \tilde{K}\}} h\left(\cdots, S_{i} \exp -\nu_{i}, \cdots\right) \exp -\delta(\nu)
$$

and its transformation

$$
\hat{h}(y)=\sup _{\{\nu \in \tilde{K}\}} h\left(\cdots, \exp \left(y_{i}-\nu_{i}\right), \cdots\right) \exp -\delta(\nu)
$$

We make on $\hat{h}(S)$ the same growth assumption as for $h(S)$, see $(2.20)$, formulated on $\hat{h}(y)$, as follows

$$
0<\hat{h}(y) \leq c\left(1+\exp \beta_{0}|y|\right)
$$


Introduce next the function $\tilde{v}(y, t)$ solution of the Cauchy problem

$$
\begin{aligned}
& \frac{\partial \tilde{v}}{\partial t}-r(t) \tilde{v}+\left(r(t)-\frac{1}{2} a_{i i}(t)\right) \frac{\partial \tilde{v}}{\partial y_{i}}+\frac{1}{2} a_{i j}(t) \frac{\partial^{2} \tilde{v}}{\partial y_{i} \partial y_{j}}=0 \\
& \tilde{v}(y, T)=\hat{h}(y)
\end{aligned}
$$

which is, with the notation $(3.30),(3.31)$,

$$
\tilde{v}(y, t)=v(y, t ; \hat{h})
$$

We check easily that

$$
\hat{h} \text { is lower semicontinuous }
$$

We state the

Lemma 4.3. We assume (2.2), (2.3), and (4.26), then one has the property

$$
D \tilde{v}(y, t) . \nu+\tilde{v}(y, t) \delta(\nu) \geq 0, \forall y, \quad t<T, \quad \forall \nu \in \tilde{K}
$$

\section{PROOF:}

Let $\theta>0$, and $\nu, \nu^{0} \in \tilde{K}$. By definition of $\hat{y}$, and for any $\mu$ we have

$$
\hat{h}(y+\theta \nu) \geq h\left(\cdots, \exp \left(y_{i}+\theta \nu_{i}-\mu_{i}\right), \cdots\right) \exp -\delta(\mu) .
$$

Since

$$
\mu=\nu^{0}+\theta \nu
$$

belongs to $\tilde{K}$, we can assert that we can deduce

$$
\hat{h}(y+\theta \nu) \geq h\left(\cdots, \exp \left(y_{i}-\nu_{i}^{0}\right), \cdots\right) \exp -\delta\left(\nu^{0}\right) \exp -\delta(\theta \nu) .
$$

hence, since $\nu^{0}$ is arbitrary in $\tilde{K}$,

$$
\hat{h}(y+\theta \nu) \geq \hat{h}(y) \exp -\delta(\theta \nu) .
$$

This can be written as

$$
\hat{h}(y+\theta \nu)-\hat{h}(y)+\hat{h}(y)(1-\exp -\theta \delta(\nu)) \geq 0 .
$$

Now , we apply Theorem (4.1) to write the probabilistic representation

$$
\tilde{v}(y, t)=E^{0} \hat{h}\left(y_{y, t}(T)\right) \gamma_{0}(t, T)
$$


therefore also

$$
\tilde{v}(y+\theta \nu, t)=E^{0} \hat{h}\left(y_{y+\theta \nu, t}(T)\right) \gamma_{0}(t, T)
$$

Since

$$
y_{y+\theta \nu, t}(T)=y_{y, t}(T)+\theta \nu
$$

we may apply $\left(4.31\right.$, with $y$ changed by $y_{y, t}(T)$ to obtain

$$
\tilde{v}(y+\theta \nu, t)-\tilde{v}(y, t)+\tilde{v}(y, t)(1-\exp -\theta \delta(\nu)) \geq 0 .
$$

Dividing by $\theta$ and letting $\theta$ tend to 0 , making use of the differentiability of $\tilde{v}$, we obtain the property (4.30) .

Remark 4.4. It follows from (3.3)and (4.30), noting that, thanks to the assumption $(4.26) \tilde{v}(y, t)>0$, the property

$$
\frac{D \tilde{v}(y, t)}{\tilde{v}(y, t)} \in K, \forall y, t
$$

We then deduce the property

Proposition 4.5. Under the assumptions of Lemma (4.3), one has the property

$$
\tilde{v}(y, 0)=v(y, 0, \hat{h}) \geq h_{u p}(K)
$$

\section{PROOF:}

From equation (4.27), one checks easily that, recalling the process $y(s)$, see $(4.20)$

$$
d\left(\tilde{v}(y(s), s) \gamma_{0}(s)\right)=\gamma_{0}(s) D \tilde{v}(y(s), s) . \sigma(s) d w^{0}(s)
$$

and thus, for $t<T$, one has

$$
\tilde{v}(y(t), t) \gamma_{0}(t)=\tilde{v}(y, 0)+\int_{0}^{t} \gamma_{0}(s) D \tilde{v}(y(s), s) \cdot \sigma(s) d w^{0}(s)
$$

and also

$$
E^{0}\left(\tilde{v}(y(t), t) \gamma_{0}(t)\right)^{2}=(\tilde{v}(y, 0))^{2}+E^{0} \int_{0}^{t}\left(\gamma_{0}(s)\right)^{2}\left|\sigma^{*}(s) D \tilde{v}(y(s), s)\right|^{2} d s
$$


Using Corollary (4.2), we can let $t$ tend to $T$ in the preceding equation, and obtain

$$
E^{0}\left(\hat{h}(y(T)) \gamma_{0}(T)\right)^{2}=(\tilde{v}(y, 0))^{2}+E^{0} \int_{0}^{T}\left(\gamma_{0}(s)\right)^{2}\left|\sigma^{*}(s) D \tilde{v}(y(s), s)\right|^{2} d s .
$$

We may then set

$$
\tilde{\pi}(s)=D \tilde{v}(y(s), s)
$$

and, recall (2.16)

$$
X_{\tilde{v}(y, 0)}^{\tilde{\pi}}(s)=\tilde{v}(y(s), s) .
$$

Thanks to (4.35) and (4.40) one may assert that

$$
\tilde{\pi} \in A(\tilde{v}(y, 0) ; K)
$$

and

$$
X_{\tilde{v}(y, 0)}^{\tilde{\pi}}(T)=\hat{h}(y(T)) \geq h(y(T)) .
$$

Therefore (4.36) follows.

From (4.36) and (3.20) we deduce

$$
\tilde{v}(y, 0)=v(y, 0, \hat{h}) \geq h_{u p}(K) \geq \hat{u}
$$

We can also check directly the inequality

$$
\tilde{v}(y, 0) \geq \hat{u}
$$

PROOF:

First recall Lemma 3.1, and equation (3.16). We can write

$$
\begin{aligned}
& u_{\nu}=E^{\nu}\left[h \left(\cdots, \exp \left(y_{i}+\int_{0}^{T}\left(r(s)-\frac{1}{2} a_{i, i}(s)\right) d s\right.\right.\right. \\
& \left.\left.+\int_{0}^{T} \sigma_{i, j}(s) d w^{\nu}(s)-\int_{0}^{T} \nu_{i}(s) d s, \cdots\right) \gamma_{\nu}(T)\right]
\end{aligned}
$$

But

$$
\int_{0}^{T} \delta(\nu(s)) d s \geq \delta\left(\int_{0}^{T} \nu(s) d s\right)
$$


Since

$$
\int_{0}^{T} \nu(s) d s \in \tilde{K}
$$

we can state

$$
u_{\nu} \leq E^{\nu}\left[\hat{h}\left(\cdots, \exp \left(y_{i}+\int_{0}^{T}\left(r(s)-\frac{1}{2} a_{i, i}(s)\right) d s+\int_{0}^{T} \sigma_{i, j}(s) d w^{\nu}(s), \cdots\right) \gamma_{0}(T)\right]\right.
$$

which can also be written

$$
u_{\nu} \leq E^{0}\left[\hat{h}\left(\cdots, \exp \left(y_{i}+\int_{0}^{T}\left(r(s)-\frac{1}{2} a_{i, i}(s)\right) d s+\int_{0}^{T} \sigma_{i, j}(s) d w^{0}(s), \cdots\right) \gamma_{0}(T)\right]\right.
$$

which means

$$
u_{\nu} \leq \tilde{v}(y, 0)
$$

and thus (4.42) follows.

We can also give a proof more in line with the standard Dynamic Programming argument. Consider

$$
\begin{aligned}
d\left(\tilde{v}(y(s), s) \gamma_{\nu}(s)=\right. & -\gamma_{\nu}(s)(D \tilde{v}(y(s), s) \cdot \nu(s)+\tilde{v}(y(s), s) \delta(\nu(s))) d s+ \\
& +\gamma_{\nu}(s) D \tilde{v}(y(s), s) \cdot \sigma(s) d w^{\nu}(s)
\end{aligned}
$$

then from (4.30) we deduce

$$
d\left(\tilde{v}(y(s), s) \gamma_{\nu}(s) \leq \gamma_{\nu}(s) D \tilde{v}(y(s), s) \cdot \sigma(s) d w^{\nu}(s) .\right.
$$

Therefore, integrating and using (4.40) as well as the continuity of $\tilde{v}(y(s), s)$, as $s \uparrow T$ it follows

$$
\begin{gathered}
\tilde{v}(y, 0) \geq E^{\nu}\left(\hat{h}\left(\cdots, \exp y_{i, y, 0}(T), \cdots\right) \gamma_{\nu}(T)\right) \\
\quad \geq E^{\nu}\left(h\left(\cdots, \exp y_{i, y, 0}(T), \cdots\right) \gamma_{\nu}(T)\right)=u_{\nu}
\end{gathered}
$$

and thus we recover again the property (4.42). 


\subsection{THE REVERSE INEQUALITY}

The objective here is to prove the reverse inequality

$$
\tilde{v}(y, 0) \leq \hat{u}
$$

which will imply from (4.41) the property (3.19), and complete the proof of Theorem 3.2.

We shall use the function, which extends (4.25)

$$
\hat{v}(y, t)=\sup _{\{\nu \in \tilde{K}\}} v(y-\nu, t) \exp -\delta(\nu)
$$

Of course $\hat{v}(y, t)$ is different from $\tilde{v}(y, t)$. However, we can state the property

$$
\hat{v}(y, t) \leq \tilde{v}(y, t) \leq C\left(1+\exp \beta_{0}|y|\right)
$$

PROOF of (4.48):

The second inequality is just a consequence of Theorem (4.1), see (4.7), and assumption (4.26). To prove the first one, we notice that

$$
\begin{gathered}
\left.v(y-\nu, t) \exp -\delta(\nu)=E^{0} h\left(y_{y, t}(T)-\nu\right)\right) \exp -\delta(\nu) \gamma_{0}(t, T) \\
\leq E^{0} \hat{h}\left(y_{y, t}(T)\right) \gamma_{0}(t, T)=\tilde{v}(y, t) .
\end{gathered}
$$

Since the supremum in (4.47) may not be attained, we use an approximation as follows

$$
\hat{v}^{\epsilon}(y, t)=\sup _{\{\nu \in \tilde{K}\}}\left[v(y-\nu, t) \exp -\delta(\nu)-\frac{\epsilon}{2}|\nu|^{2}\right]
$$

Then we can state the property: there exists $\hat{\nu}^{\epsilon}(y, t)$, measurable, such that

$$
\hat{v}^{\epsilon}(y, t)=v\left(y-\hat{\nu}^{\epsilon}, t\right) \exp -\delta\left(\hat{\nu}^{\epsilon}\right)-\frac{\epsilon}{2}\left|\hat{\nu}^{\epsilon}\right|^{2}
$$

for any $y \in R^{n}, t<T$.

PROOF of (4.50):

Consider the function

$$
\Phi^{\epsilon}(y, t ; \nu)=v(y-\nu, t) \exp -\delta(\nu)-\frac{\epsilon}{2}|\nu|^{2}
$$


which is continuous in $y, t$ and u.s.c. in $\nu$. To find the supremum, we can restrict $\nu$ so that

$$
\frac{\epsilon}{2}|\nu|^{2} \leq v(y-\nu, t) \exp -\delta(\nu) \leq \hat{v}(y, t)
$$

and from (4.48)

$$
\frac{\epsilon}{2}|\nu|^{2} \leq C\left(1+\exp \beta_{0}|y|\right) .
$$

Consider the ball $B_{R}$, of center 0 and radius $R$, and the function $\Phi^{\epsilon}(y, t ; \nu)$, restricted to $B_{R} \times(0, T)$. Then the supremum is obtained on the compact set

$$
\frac{\epsilon}{2}|\nu|^{2} \leq C\left(1+\exp \beta_{0} R\right) .
$$

Therefore, using classical selection properties, see I. EKELAND, R.TEMAM [1], there exists a selection $\hat{\nu}_{R}^{\epsilon}(y, t)$, such that

$$
\hat{v}^{\epsilon}(y, t)=v\left(y-\hat{\nu}_{R}^{\epsilon}, t\right) \exp -\delta\left(\hat{\nu}_{R}^{\epsilon}\right)-\frac{\epsilon}{2}\left|\hat{\nu}_{R}^{\epsilon}\right|^{2}
$$

for any $y \in B_{R}, t<T$.

If we define

$$
\hat{\nu}^{\epsilon}(y, t)=\sum_{\{n=1\}}^{+\infty} \mathbb{1}_{B_{n}-B_{n-1}}(y) \hat{\nu}_{n}^{\epsilon}(y, t)
$$

this function satisfies the properties.

We then define

$$
\hat{\nu}_{t}^{\epsilon}(s)=\left\{\begin{array}{l}
0 \text { if } s<t \\
\frac{\hat{\nu}^{\epsilon}(y(t), t)}{T-t}, \text { if } s \geq t
\end{array}\right.
$$

We can check that

$$
\hat{\nu}_{t}^{\epsilon} \in \mathcal{D}^{\{m\}} .
$$

Indeed, considering $Z_{\hat{\nu}_{t}^{\epsilon}}(s)$, then one has

$$
\begin{aligned}
& Z_{\hat{\nu}_{t}^{\epsilon}}(s)=1, \quad \text { if } s<t \\
& Z_{\hat{\nu}_{t}^{\epsilon}}(s)=\exp \left[-\frac{\left(\hat{\nu}^{\epsilon}\right)^{*}(y(t), t)}{T-t} \int_{t}^{s}\left(\sigma^{-1}\right)^{*} d w^{0}\right. \\
& \left.-\frac{1}{2} \frac{\left(\hat{\nu}^{\epsilon}\right)^{*}(y(t), t)}{T-t} \int_{t}^{s} a^{-1}(\tau) d \tau \frac{\hat{\nu}^{\epsilon}(y(t), t)}{T-t}\right]
\end{aligned}
$$


Therefore, one has, as easily seen

$$
E^{0}\left[Z_{\hat{\nu}_{t}^{\epsilon}}(T) \mid \mathcal{F}^{t}\right]=1
$$

and the martingale property follows .

We can then assert that

$$
\hat{u} \geq u_{\hat{\nu}_{t}^{\epsilon}} \text {. }
$$

But we have, see (4.43)

$u_{\hat{\nu}_{t}^{\epsilon}}=E^{\hat{\nu}_{t}^{\epsilon}}\left[h\left(y(t)+\rho(t, T)+\int_{t}^{T} \sigma(s) d w^{\hat{\nu}_{t}^{\epsilon}}(s)-\hat{\nu}^{\epsilon}(y(t), t)\right) \gamma_{0}(T) \exp -\delta\left(\hat{\nu}^{\epsilon}(y(t), t)\right)\right]$.

Taking first the conditional expectation with respect to $\mathcal{F}^{t}$ yields

$$
u_{\hat{\nu}_{t}^{\epsilon}}=E^{0}\left[v\left(y(t)-\hat{\nu}^{\epsilon}(y(t), t)\right) \gamma_{0}(t) \exp -\delta\left(\hat{\nu}^{\epsilon}(y(t), t)\right)\right]
$$

and recalling the definition of $\hat{\nu}^{\epsilon}(y, t)$, we can write

$$
u_{\hat{\nu}_{t}^{\epsilon}} \geq E^{0} \hat{v}^{\epsilon}(y(t), t) \gamma_{0}(t)
$$

But, it is easy to check that

$$
v^{\epsilon}(y, t) \uparrow v(y, t)
$$

By Fatou's Lemma, we deduce

$$
\hat{u} \geq E^{0} \hat{v}(y(t), t) \gamma_{0}(t)
$$

Now, we let $t \uparrow T$, in the right hand side of (4.56). By Fatou's Lemma again, we state

$$
\hat{u} \geq E^{0} \liminf _{t \uparrow T} \hat{v}(y(t), t) \gamma_{0}(T)
$$

But

$$
\begin{gathered}
\liminf _{t \uparrow T} \hat{v}(y(t), t) \geq \lim _{t \uparrow T} v(y(t)-\nu, t) \exp -\delta(\nu), \forall \nu \\
\geq h\left(\cdots, \exp \left(y_{i}(T)-\nu_{i}\right), \cdots\right) \exp -\delta(\nu), \forall \nu
\end{gathered}
$$

hence

$$
\liminf _{t \uparrow T} \hat{v}(y(t), t) \geq \hat{h}\left(\cdots, \exp y_{i}(T), \cdots\right) .
$$

From (3.30), it then follows

$$
\hat{u} \geq E^{0} \hat{h}\left(\cdots, \exp y_{i}(T), \cdots\right) \gamma_{0}(T)=\tilde{v}(y, 0) .
$$

which is (4.46). 


\section{CHARACTERIZATION OF THE LOWER HEDGING PRICE}

We just state the counterpart of the previous section. Setting, to simplify the notation

$$
K=K_{-}
$$

and defining successively

$$
\begin{aligned}
& \zeta(\nu)=\inf _{p \in K}\left(-p^{*} \nu\right) \\
& \tilde{K}=\{\nu \mid \zeta(\nu)>-\infty\} \\
& \check{h}(y)=\inf _{\{\nu \in \tilde{K}\}} h(y-\nu) \exp -\zeta(\nu) \\
& \bar{v}(y, t)=E^{0} \check{h}\left(y_{y, t}(T)\right) \gamma_{0}(t, T)=v(y, t ; \check{h}) \\
& \check{u}=\inf _{\left\{\nu(.) \in \mathcal{D}^{(m)}\right\}} E^{\nu}\left(h(y(T)) \gamma_{\nu}(T)\right.
\end{aligned}
$$

then we have the property

$$
\check{u}=h_{\text {low }}(K)=\bar{v}(y, 0)
$$

\section{References}

[1] I. EKELAND, R. TEMAM, Convex Analysis and Variational Problems, North Holland, Amsterdam (1976)

[2] A. FRIEDMAN, Partial Differential Equations of parabolic type, Prentice Hall, N.J. (1964)

[3] N. IKEDA, S. WATANABE, Stochastic Differential Equations and Diffusion Processes North Holland, Amsterdam (1981)

[4] I. KARATZAS, S.G. KOU, On the Pricing of Contingent Claims with Constraints, The Annals of Applied Probability, Vol 6, N 2, pp 321-369

[5] I. KARATZAS, S.E. SHREVE, Methods of Mathematical Finance, Applications of Mathematics, Springer Verlag, New York (1998)

[6] J. L. LIONS, Contrôle optimal des systèmes gouvernés par des équations aux dérivées Dunod, Paris (1969)

[7] R.T. ROCKAFELLAR, Convex Analysis, Princeton University Press, Princeton, N.J. 